However, even well developed evidencebased guidelines are often not used in day-today care (estimations range from $25-50 \%$ ). There are different causes for clinical guidelines not being used, partly related to the guidelines themselves. For instance, some guidelines are written as a handbook on a clinical topic and not as a concise set of concrete recommendations for decisions in day-to-day practice. They often have too many recommendations, making it difficult for the user to identify the key-issues and most important targets. Many do not answer the crucial questions of patients and practitioners related to the health problem well, as outlined by Hegarty et al. ${ }^{3}$

In a large study (yet unpublished) on the implementation of chronic obstructive pulmonary disease (COPD) guidelines in the Netherlands, we found that improvement failed, largely because patients did not follow the advice of their GPs. The guidelines on COPD do not address this problem of nonadherence sufficiently. Many guidelines also lack the tools that should help to make them work in real practice, such as well-structured care pathways and well-developed indicators to measure performance and change, and focused programmes to support their implementation.

Clinical guidelines are potentially very valuable tools to support decision making in general practice, but some improvements are required in current guidelines and guideline development processes to make them more relevant. They should, for instance, be focused more on key-issues in patient care, with direct relevance for both practitioners and patients; they should take real (comorbid) patients as a starting point; they should be developed in less time-intensive procedures to keep them updated, presented in more concise formats, and be combined with quality indicators and support tools for practice. A priority is better collaboration between all stakeholders clinicians, scientists, patients, policymakers, and others - to identify jointly the most important questions, assess the available evidence, and draw recommendations that can work under prevailing practice conditions. ${ }^{11}$ The limitations and importance of drawing guidelines for highly different circumstances under which practitioners encounter their patients should be acknowledged. This is even more important when financial incentives are linked to the evidence-based guidelines. Such improvements should lead to guidelines being able to deliver what they intend: better care for patients in response to their needs.

\section{Richard Grol,}

Director, IQ Healthcare, Radboud University Nijmegen Medical Centre, Nijmegen, the Netherlands.

\section{Chris van Weel,}

Chair, Department of Family Medicine, Radboud University Medical Centre, Nijmegen,

the Netherlands.

\section{REFERENCES}

1. Burgers JS, Cluzeau FA, Hanna SE et al. Characteristics of high-quality guidelines: evaluation of 86 clinical guidelines developed in ten European countries and Canada. Int J Technol Assess Health Care 2003; 19(1): 148-157.

2. Van Roosmalen MS, Braspenning JC, De Smet PA, Grol RP. Antibiotic prescribing in primary care: first choice an restrictive prescribing are two different traits. Qual Saf

Health Care 2007; 16(2): 105-109.

3. Hegarty K, Gunn J, Blashki G, et al. How could depression guidelines be made more relevant and applicable to primary care? A quantitative and qualitative review of national guidelines. Br J Gen Pract 2009; 59: 322-328.

4. Burgers JS, Bailey JV, Klazinga NS, et al. Inside guidelines: comparative analysis of recommendations and evidence in diabetes guidelines from 13 countries. Diabetes Care 2002; 25(11): 1933-1939.

5. Wennekes L, Hermens RP, van Heumen K, et al. Possibilities for transborder cooperation in breast cancer care in Europe: a comparative analysis regarding the content, quality and evidence use of breast cancer guidelines. Breast 2008; 17(5): 464-471.

6. Wollersheim H. Beyond the evidence of guidelines. Neth Med 2009; 67(2): 39-40.

7. De Kort S, Burgers J, Willems D. Value judgements that matter to patients remain implicit in oncology guidelines: an observational study. Neth J Med 2009; 67(2): 62-68.

8. Van Weel C, Knottnerus JA. Evidence-based interventions and comprehensive treatment. Lancet 1999; 353(9156): 916-918.

9. Van Weel C, van Weel-Baumgarten E, van Rijswijk E. Treatment of depression in primary care. BMJ 2009; 338: b934.

10. Dowrick C, Leydon GM, McBride A, et al. Patients' and doctors' views on depression severity questionnaires incentivised in UK quality and outcomes framework: qualitative study. BMJ 2009; 338: b663.

11. Schünemann HJ, Woodhead M, Anzueto A, et al. A vision statement on guideline development for respiratory disease: the example of COPD. Lancet 2009; 373(9665): 774-779.

DOI: 10.3399/bjgp09X420554

\section{ADDRESS FOR CORRESPONDENCE}

\section{Richard Gro}

Scientific Institute for Quality of HealthCare, Radboud University Nijmegen, PO Box 9101, 114 IQ Healthcare, Nijmegen, 6500 HB, Netherlands. E-mail: r.grol@iq.umcn.nl

\title{
Direct access to diagnostic services
}

Under conventional systems of care, outpatient clinics see patients referred by a GP for clinical assessment by a hospital specialist. Subsequent hospital visits are arranged to undertake any specialist diagnostic tests that may be required and to initiate treatment where necessary. In other words, the specialist in the outpatient clinic acts as a gatekeeper to other hospital resources. Allowing the GP to bypass this gatekeeper and gain 'direct access' to tests can enable GPs to make more efficient use of hospital resources and reduce waiting times for patients.

Direct access to diagnostic services should reduce outpatient attendance in that GPs may refer patients for diagnostic testing without prior consultant assessment. Waiting time from presentation to testing is accordingly reduced. If the patient can be managed by the GP without subsequent referral to a consultant, waiting time from presentation to treatment is also reduced and further outpatient attendance avoided. However, direct access may increase demand for testing and lead to less appropriate referrals with a consequent reduction in diagnostic yield. It is also possible that the quality of care will decline if GPs fail to take appropriate clinical action in response to test results. All other factors being equal, the direct cost to hospitals may be reduced if savings from reduced referral rates to outpatient clinics are greater than the costs of providing the direct access service. 
A scoping review of research in 2007 identified 27 evaluations of direct access to diagnostic services, spanning echocardiography, electrocardiography, gastroscopy, sigmoidoscopy and ultrasound. ${ }^{1,2}$ The quality of studies was generally poor with only two of the 27 employing a (quasi) experimental design and the rest being observational in nature. Subsequently, two high-quality randomised trials of direct access have been published. The DAMASK trial examined direct access for magnetic resonance imaging (MRI) of the knee., ${ }^{3,4}$ The OATS trial, reported in this issue of the $B J G P$, evaluates hysterosalpingography for the investigation of primary infertility. ${ }^{5,6}$

Previous research suggests that the principle benefit for patients of direct access to diagnostic services is reduced waiting time from presentation to testing and hence treatment. Both the DAMASK and OATS trials echo these findings in showing markedly shorter waiting times for those patients whose GPs used direct access. However, as few GPs opted to use the service in the OATS trial, there was no measurable benefit to the eligible patient population as a whole. The accompanying qualitative research revealed that GPs may have failed to take full advantage of direct access because infertility presents rarely and is not life threatening; hence GPs felt poorly prepared and motivated to take on the task of full initial assessment. If this were the complete story then we might expect to have seen a high uptake of direct access by GPs in the DAMASK trial, as knee problems are prevalent in general practice and GPs are familiar with their management. However, DAMASK recruited only 553 patients from 163 practices over a 1-year period suggesting that GPs were reluctant to take over patient investigation from specialists.

The OATS study agrees with past research in finding that patient and professional satisfaction with direct access is generally high. While some patients will always wish to bypass general practice and go directly to a specialist, most welcome initial investigation by their GP. GPs themselves like having the option of direct access even if they elect not to use it. Hospital specialists prefer seeing patients who have already undergone all preliminary tests and investigations, as this makes more efficient use of their time.
The principle risk of direct access is that GPs will make inappropriate use of the service and push up demand on hospital diagnostic services. Previous research suggests these concerns are unfounded with GPs using investigations as appropriately as specialists. ${ }^{1,2}$ Hence where demand rises, it is targeted to unmet clinical need. The OATS study found that the completeness of information on referral was higher when GPs had used direct access hysterosalpingography, aided by the use of clear guidelines embedded in test order forms. In other words, research is consistent in suggesting that those GPs who elect to use a direct access diagnostic test do so appropriately.

Research further suggests that direct access to diagnostic testing allows GPs to manage a substantial number of patients who would otherwise be referred to outpatient clinics. While there is a risk that GPs may fail to act appropriately on a test result, there has been a paucity of research into this issue. The DAMASK trial found a slightly higher quality of life, but no objective clinical differences, in patients who received direct access MRI for knee problems in primary care. The OATS study, like most of its predecessors, does not make clear whether GPs' subsequent management decisions were appropriate. Neither DAMASK nor OATS quantify how many inappropriate referrals to secondary or tertiary care services were avoided as a result of direct access investigation. Such issues warrant investigation in future.

Previous research into healthcare costs is sketchy but suggests that savings in reduced attendance at outpatient clinics may offset any increase in the cost to hospital diagnostic services of providing direct access. The DAMASK trial found that costs increased with direct access MRI but so too did patients' quality of life, making it a cost-effective use of resources. The OATS study provides no economic information but savings (or increases) are likely to have been small given the low uptake by GPs. The cost implications of providing direct access to diagnostic services merits particular attention in future research as it plays a critical part in the commissioning of health services.

In summary, direct access to diagnostic services can enable GPs to make more efficient use of hospital resources and reduce patient waiting times. But these gains are likely to be achieved only if GPs make full use of the new services. More research is needed into the factors limiting GP uptake of direct access services, and how these may be overcome, if the full benefits for patients are to be realised.

\section{Bonnie Sibbald,}

Professor of Health Services Research and Deputy Director, National Primary Care Research and Development Centre, University of Manchester, Manchester.

\section{REFERENCES}

1. Sibbald B, McDonald R, Roland M. Shifting care from hospitals to the community: a review of the evidence on quality and efficiency. J Health Serv Res Policy. 2007; 12(2): 110-117.

2. Roland M, McDonald R, Sibbald B. Chapter 3: transfer to primary care. In: Outpatient services and primary care. A scoping review of research into strategies for improving outpatient effectiveness and efficiency. Manchester: National Primary Care Research and Development Centre, 2006.

http://www.npcrdc.ac.uk/PublicationDetail.cfm?id=156 (accessed 2 Apr 2009).

3. DAMASK (Direct Access to Magnetic Resonance Imaging: Assessment for Suspect Knees) Trial Team.

Effectiveness of GP access to magnetic resonance imaging of the knee: a randomised trial. Br J Gen Pract 2008; DOI: 10.3399/bjgp08X342651.

4. DAMASK (Direct Access to Magnetic Resonance Imaging: Assessment for Suspect Knees) Trial Team. Costeffectiveness of magnetic resonance imaging of the knee for patients presenting in primary care. Br J Gen Pract 2008; DOI: $10.3399 /$ bjgp 08x342660.

5. Wilkes S, Murdock A, Steen N, et al. Open Access Tubal aSsessment for the initial management of infertility in general practice (the OATS trial): a pragmatic cluster randomised controlled trial. Br J Gen Pract 2009; 59 (562): 329-335.

6. Wilkes S, Rubin G, Crossland A, et al. Patient and professional views of open access hysterosalpingography (HSG) for the initial management of infertility in primary care. Br J Gen Pract 2009; 59 (562): 336-342.

DOI: 10.3399/bjgp09X420563

ADDRESS FOR CORRESPONDENCE

\section{Bonnie Sibbald}

National Primary Care Research, and Development Centre, University of Manchester, 5th Floor, Williamson Building, Oxford Road, Manchester, M13 9PL. E-mail: Bonnie.Sibbald@manchester.ac.uk 